\title{
OBJECT IDENTIFICATION BY USING ORTHONORMAL CIRCUS FUNCTIONS FROM THE TRACE TRANSFORM
}

\author{
Andrés Frías-Velázquez*, Carlos Ortiz ${ }^{\dagger}$, Aleksandra Pižurica*, Wilfried Philips* and Gustavo Cerda \\ *IPI-TELIN-UGent/IBBT, Sint-Pietersnieuwstraat, B-9000 Ghent, Belgium \\ ${ }^{\dagger}$ DICIS - U. de Guanajuato, Carr. Salamanca-Valle km 3.5+1.8, 36700 Salamanca, México. \\ E-mail: Andres.FriasVelazquez@telin.ugent.be
}

\begin{abstract}
In this paper we present an efficient way to both compute and extract salient information from trace transform signatures to perform object identification tasks. We also present a feature selection analysis of the classical trace-transform functionals, which reveals that most of them retrieve redundant information causing misleading similarity measurements. In order to overcome this problem, we propose a set of functionals based on Laguerre polynomials that return orthonormal signatures between these functionals. In this way, each signature provides salient and non-correlated information that contributes to the description of an image object. The proposed functionals were tested considering a vehicle identification problem, outperforming the classical trace transform functionals in terms of computational complexity and identification rate.
\end{abstract}

Index Terms - Object identification, Trace transform, Orthonormal signatures, Laguerre polynomials, Feature selection.

\section{INTRODUCTION}

The Trace transform is a radial projection transformation that allows one to construct image features that are invariant to a chosen group of image transformations [1]. The trace transform can be considered as a generalization of the Radon transform, which is widely used in computational tomography. The main object of the trace transform is to obtain image descriptors such as the sinogram, circus function, or triple feature to perform object recognition tasks. Such descriptors are computed in a cascade fashion from the trace transform, reducing its dimensionality at each processing stage. In short, trace transform descriptors are obtained by applying a functional, called trace or $T$-functional, over the tracing lines of an image at different angular orientations. The resulting image is referred to as sinogram, which in turn is a transformation from the euclidean space $(x, y)$ into a Hough space $(p, \theta)$. The sinogram can be further processed by applying another functional, called diametrical or $P$-functional, over the radial coordinate returning a 1-D signature called circus function. Finally, the description of an image can be still reduced by applying another functional, referred to as $\Phi$-functional, over the circus function returning a single value, known as triple feature.

Applications based on the trace transform span over several object recognition and identification tasks such as face identification [2, $3]$, human action recognition [4], image retrieval [5, 6], as well as parameter estimation of geometrical transformations [7]. Previous

This work has been supported by the Flemish Interdisciplinary Institute for Broadband Technology (IBBT) and by the Flemish Fund for Scientific Research. works have shown the robustness of the trace transform under different image variations such as affine geometrical distortions, uneven illumination, noise perturbations and occlusions. However, it has not been extensively exploited due to its high computational complexity, as discussed in [8]. The main inconvenience lies in the large number of functionals applied over the tracing lines at different orientations of the image, which demands significant processing power. Note that most of the aforementioned literature showed very good recognition results at the expense of a high computational complexity. For instance, Kadyrov and Petrou [9] employed 42 circus functions in order to characterize an image in an exercise of fish recognition. On the other hand, Srisuk et al. [2] proposed a face authentication system based on 22 trace transform sinograms, where their relevant edges were used as descriptors. As a reference of the system's time complexity, this paper reports that the feature extraction and training process of 2360 faces takes $100 \mathrm{~h}$, while every image test took about 30 s.

In this paper we present a statistical analysis, which concludes that some of the functionals widely used in the literature convey very similar information. As a result, they can be considered redundant, since they really do not contribute with new evidence to discriminate between images. Likewise, such redundant features represent a burden for the system from a computational point of view. In order to overcome these problems, we propose a set of orthonormal functionals based on Laguerre polynomials in order to derive orthonormal circus functions. The idea is to generate non-correlated descriptors that contribute independently to the decision whether two image objects are similar or not. In this way, we can increase the identification performance and reduce the computational overhead at the same time.

The paper is organized as follows. In section 2, a feature selection analysis of the classical trace transform functionals is presented. In section 3, we describe the procedure to derive orthonormal circus functions from Laguerre polynomials. Section 4 evaluates the identification performance of the orthonormal circus functions. Finally, the conclusions of this work are stated in section 5 .

\section{FEATURE SELECTION ANALYSIS OF THE TRACE TRANSFORM SIGNATURES}

When different features are extracted from an image to perform object identification tasks, we expect such descriptors to be uncorrelated to each other. In this way, we can match image pairs described with very similar features and discriminate among other candidate images. For the trace-transform features, the independence among circus functions is assumed by using different functionals. However, in reality, such assumption does not always hold, which may yield 


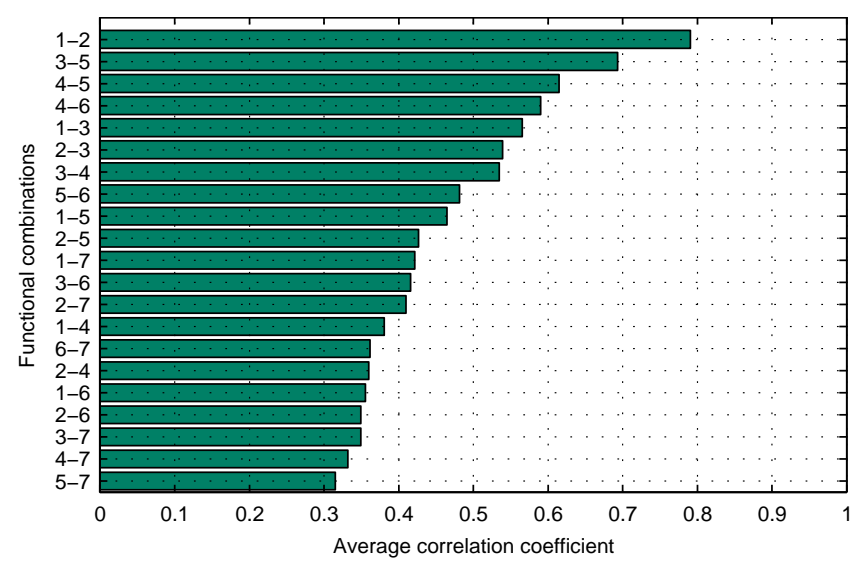

Fig. 1. Correlation among different $T$-functionals.

highly correlated circus functions. As a result, several of these signatures may carry superfluous information and provide little benefit to the identification performance.

Note that in previous works, the features extracted from the trace transform for classification or identification problems aimed to obtain features invariant to geometrical distortions. Nevertheless, little was known about their actual discriminative power. For instance, in the seminal paper of Kadyrov and Petrou [5] several ways to construct functionals that comply with the affine invariance conditions were presented. However, there is no guarantee that a given set of those functionals provide uncorrelated features, and thus sufficient discriminatory information. As a result, feature selection methods may shed some light on how to choose the most important functionals in order to reduce their number and at the same time retain as much as possible the discriminatory information.

In order to evaluate the dependency between functionals proposed in the literature, we performed a feature selection analysis based on the filter approach. The filter approach is a combinatorial optimization method that attempts to find the best feature combinations from a pool of choices by evaluating an optimality criteria. A review of several feature selection methods can be found in [10].

A feature selection analysis was performed to evaluate the signatures' correlation coefficient resulting from the $T$-functionals and $P$-functionals presented in Table 1 and 2, respectively. Note that in these tables, $f(t)$ is the signal along the tracing line of the parameter $t$, while $g(p)$ represents a diametric trace line of the sinogram $G(p, \theta)$. The assessment of these functionals was performed with a vehicle image database which contains 1131 exemplars. For each image we computed $7 T$-funct. $\times 3 P$-funct. $=21$ circus functions.

In Fig. 1, we present the average correlation coefficient between circus functions derived from each possible pair of $T$-functionals. From this figure, we can readily notice that the correlation of the signatures derived from the $T$-functionals 1 and 2 is the largest. This result suggests that these two functionals convey very similar information. Also, the pairs resulting from the $T$-functionals 3,4 and 5 seem to convey redundant information. Note that each group of functionals mentioned above has similar weighting kernels. As a result, we may conclude that the signatures' dependency may be largely influenced by the weighting kernel of the functional. Conversely, the combination of functionals whose weighting kernel is different (e.g., 2, 5 and 7) returned lower correlations, and thus less redundancy.

A similar statistical analysis was performed for the $P$-functionals. The outcome of this test revealed that the average correlation between the $P$-functionals 1 and 2 was 0.4507 , while for the Pfunctionals 1 and 3 was 0.4554 . The combination of the functionals
Table 1. List of $T$-functionals.

\begin{tabular}{|c|c|}
\hline Code & Functional \\
\hline 1 & $T(f(t))=\int_{R_{+}} r f(r) d r$ \\
\hline 2 & $T(f(t))=\int_{R_{+}}^{n_{+}} r^{2} f(r) d r$ \\
\hline 3 & $T(f(t))=\left|\int_{R_{+}} e^{i 5 \log \left(r_{1}\right)} r_{1} f\left(r_{1}\right) d r\right|$ \\
\hline 4 & $T(f(t))=\left|\int_{R_{+}} e^{i 3 \log \left(r_{1}\right)} f\left(r_{1}\right) d r_{1}\right|$ \\
\hline 5 & $T(f(t))=\left|\int_{R_{+}} e^{i 4 \log \left(r_{1}\right)} r_{1}^{0.5} f\left(r_{1}\right) d r_{1}\right|$ \\
\hline 6 & $T(f(t))=$ median $_{t_{k}>0}\left\{\left|r_{1} f\left(r_{1}\right)\right|,\left|f\left(r_{1}\right)\right|^{1 / 2}\right\}$ \\
\hline 7 & $T(f(t))=$ median $_{t_{k}>0}\left\{|f(r)|,|f(r)|^{1 / 2}\right\}$ \\
\hline \multicolumn{2}{|r|}{$\begin{array}{l}\text { Note: } r=t-c, c=\text { median }\left(\left\{t_{k}\right\}_{k},\left\{\left|f\left(t_{k}\right)\right|\right\}_{k}\right) \\
\quad r_{1}=t-c_{1}, c_{1}=\operatorname{median}\left(\left\{t_{k}\right\}_{k},\left\{\left|f\left(t_{k}\right)\right|^{1 / 2}\right\}_{k}\right)\end{array}$} \\
\hline & Table 2. List of $P$-functionals. \\
\hline \multicolumn{2}{|c|}{ Code Functional } \\
\hline 1 & $P(g(p))=\sum_{k}\left|g\left(p_{k+1}\right)-g\left(p_{k}\right)\right|$ \\
\hline 2 & $P(g(p))=\operatorname{median}\left(\left\{g\left(p_{k}\right)\right\}_{k},\left\{\left|g\left(p_{k}\right)\right|\right\}_{k}\right)$ \\
\hline & $P(g(p))=\int|\mathcal{F}\{g(p)\}(\omega)|^{4} d \omega$ \\
\hline
\end{tabular}

2 and 3 returned a correlation of 0.5382 . Consequently, all these three $P$-functionals are not highly correlated to each other, although their values are neither negligible.

The feature selection analysis shows that most of the classical functionals employed in the literature convey redundant information. Consequently, it is expected that some of those functionals can be discarded, while retaining most of the discriminatory information. In section 4 we will discuss in more detail the actual impact of the feature selection analysis on the identification performance.

\section{ORTHONORMAL CIRCUS FUNCTIONS}

As stated in section 2, the circus functions generated by uncorrelated functionals are the most salient and efficient features to describe an image object. The feature selection analysis brought to light information about the performance of the functionals, and gave some hints about how to intuitively pick good functional combinations. However, any functional combination chosen from Tables 1 and 2 is far from optimal in terms of information efficiency. In other words, no combination of functionals yields uncorrelated circus functions, as shown in Fig. 1. As a result, it is necessary to propose a set of functionals that guarantee the independence of the circus functions generated. Hence, the following condition should be fulfilled:

$$
\rho=\frac{\left\langle h_{n}(\theta), h_{m}(\theta)\right\rangle}{\left\|h_{n}(\theta)\right\|\left\|h_{m}(\theta)\right\|}=\delta(n-m)
$$

where $h_{n}(\theta)=\left\langle w_{n}(p), G(p, \theta)\right\rangle$ is the circus function obtained via the inner product between the weighting kernel $w_{n}(p)$ and the sinogram $G(p, \theta)$. Therefore, the independence between circus functions depends on both the weighting kernel and the sinogram. Recall that in the previous section we found out that the weighting kernels of classical functionals have a substantial influence on the features' saliency. For this reason, it is necessary to find a set of weighting kernels able to fulfill (1). Note that if we assume that the sinogram is orthonormal, that is $\left\langle G(p, \theta), G\left(p^{\prime}, \theta\right)\right\rangle=\delta\left(p-p^{\prime}\right)$, we can rewrite (1) as follows:

$$
\rho=\frac{\left\langle w_{n}(p), w_{m}(p)\right\rangle}{\left\|w_{n}(p)\right\|\left\|w_{m}(p)\right\|}=\delta(n-m)
$$

where the dependency among circus functions lies exclusively over the weighting kernels. In practice, in order to comply with the sino- 
gram orthonormalization condition, the nearest orthonormal matrix of the sinogram $G$ is derived as follows:

$$
\Gamma=\underset{\Omega}{\operatorname{argmin}}\|\Omega-G\|_{F} \quad \text { subject to } \quad \Omega^{T} \Omega=I
$$

where $\Omega$ is any orthonormal matrix, $\Gamma$ is the nearest orthonormal matrix, and $\|\cdot\|_{F}$ is the Frobenius norm. Schönemann proposed an efficient solution to this problem based on the singular value decomposition (SVD) [11].

In light of (2), the correlation coefficient between circus functions solely depends on the correlation of the weighting functions. Therefore, we propose a set of orthonormal weighting kernels derived from the Laguerre polynomials $L_{k}(p)$, which fulfill the orthonormality condition as follows

$$
\int_{0}^{\infty} L_{n}(p) L_{m}(p) e^{-p} d p=\delta(n-m)
$$

The polynomials can be derived by using the following recurrence relation $L_{k+1}(p)=\frac{1}{k+1}\left((2 k+1-p) L_{k}(p)-k L_{k-1}(p)\right)$, given that $L_{0}=1$ and $L_{1} \stackrel{k+1}{=} 1-p$. Note that (4) can be factorized as shown in (5), and by analogy between (2) and (5), we conclude that the orthonormal weighting kernels can be defined as in (6).

$$
\begin{gathered}
\int_{0}^{\infty}\left(L_{n}(p) e^{-p / 2}\right)\left(L_{m}(p) e^{-p / 2}\right) d p=\delta(n-m) \\
w_{n}(p)=L_{n}(p) e^{-p / 2}
\end{gathered}
$$

Finally, the diametric-orthonormal functionals can be expressed as

$$
P(g(p))=\int_{R_{+}} L_{n}(z) e^{-z / 2} \gamma(z) d z
$$

where $z=p-c_{3}$ and $c_{3}=\operatorname{median}\left(\left\{p_{k}\right\}_{k},\left\{\left|g\left(p_{k}\right)\right|\right\}_{k}\right)$, while $\gamma(z)$ is a diametric trace line of the nearest orthonormal matrix $\Gamma$. In summary, the orthonormal circus functions are generated as follows:

1. Generate the trace transform sinogram $G(p, \theta)$ of an image by using low correlated $T$-functionals.

2. Change the domain of the sinogram from $p$ to $z$.

3. Obtain the Nearest Orthonormal Sinogram (NOS) via SVD, as described in [11].

4. Apply the Laguerre $P$-functionals derived in (7) over the NOS.

It is worth to mention that the proposed functionals do not fulfill the homogeneity properties established in [1]. However, we will show in the following section that under moderate scale differences, the orthonormal functionals achieve a better identification performance than the homogeneous functionals.

\section{EXPERIMENTAL RESULTS}

In order to test the proposed improvements, we performed a vehicle identification experiment by comparing the performance of the proposed orthonormal circus functions against the classical approach proposed by Petrou and Kadyrov [1]. The aim is to get the correct correspondence between two sets of vehicle images that have been captured under different conditions. For this experiment, two corresponding images may experience translation, scale, illumination and pose variations. Some examples of the tested images are presented in Fig. 2. These images were captured from different surveillance
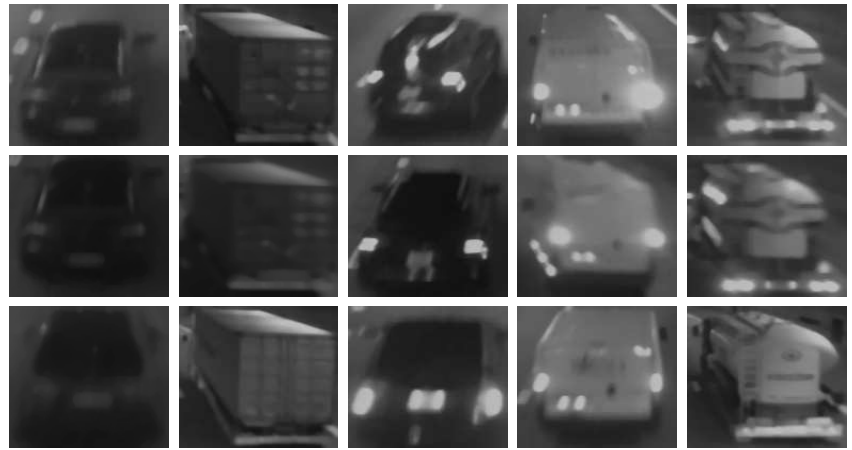

Fig. 2. Sample of vehicle images captured in a tunnel.

cameras placed in a tunnel. Their bit-depth is 8-bit gray-scale and their resolution is $85 \times 85$ pixels. The first row in Fig. 2 shows a group of vehicles that have been captured by one of the cameras in the tunnel, while the second row shows their corresponding observation registered from another camera at a different place of the tunnel. The group of vehicles found in the third row do not have any correspondence with the vehicles depicted before; however, their resemblance may lead to identification errors. Note that apart from the close similarity among vehicles, the geometrical distortions between corresponding images and the poor illumination conditions turn the vehicle identification problem into a challenging task.

The image database is composed of 377 vehicles. Each vehicle has 3 image instances taken from 3 cameras with non-overlapping view. For each image we computed $7 T$-funct. $\times 3 P$-funct. $=$ 21 circus functions derived from the combination of the well-known functionals shown in Tables 1 and 2. On the other hand, the orthonormal circus functions were computed using the $T$-functionals with the lowest correlation between them (i.e., 2, 5, 6, and 7 ) according to Fig. 1 . Then, the Laguerre $P$-functionals, defined in (7), were applied using the following polynomials $L_{1}(z)=1-z$ and $L_{2}(z)=\frac{1}{2} z^{2}-2 z+1$. As a result, 8 orthonormal circus functions were generated from the combination of $4 T$ - and $2 P$-functionals.

In Fig. 3 we present the feature selection analysis of the orthonormal circus functions for all possible $T$-functional combinations. Note that the same analysis was presented in Fig. 1, but for non-orthonormal signatures. By comparing both figures, we can verify that with the orthonormal circus functions the correlation decreased in average by $50 \%$ for the same $T$-functional combinations. On the other hand, the average correlation between signatures generated by the two Laguerre $P$-functionals was 0.1139 , which is much lower than the average of 0.4814 obtained with the $P$-functionals of Table 2. Note that despite the correlation values were not exactly zero for the orthonormal signatures, the correlation of all combinations was considerably low. This experimental-theoretical discrepancy is mainly due to finite precision errors during numerical integration, quasi-orthonormality of the NOS, as well as the weak correlation that persists among $T$-functionals.

The identification process was performed by comparing the circus functions of all possible matching combinations between two groups of vehicles captured from different cameras. In this way, a confusion matrix is created, which serves to the Hungarian algorithm to find the optimal assignment between vehicle groups. In Fig. 4, we can observe the identification performance of the circus functions derived with the classical and orthonormal circus functions. Note that the identification performance was assessed under different degrees of uncertainty by varying the size of the group of vehicles (matching window). The identification rate for each window size 


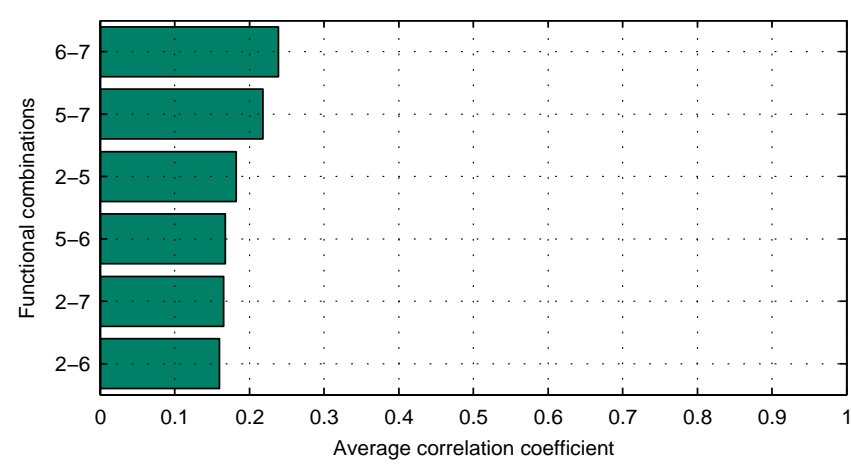

Fig. 3. $T$-functional intercorrelation with orthonormal signatures.

was computed by finding the correspondence of 50 different vehicle groups extracted from the 377 vehicles, and then averaging the identification rate of all the groups. The circle-marked line denotes the identification performance when the traditional functionals are used. The similarity between vehicles was evaluated by averaging the top 8 ranked similarity coefficients out of 21, as suggested in [9], yielding the worst performance. On the other hand, if all the 21 similarity coefficients are averaged (asterisk-marked line), the performance improves considerably. The star-marked line shows the average of the similarity coefficients derived from the $T$-functionals $2,5,6,7$ and the $P$-functionals 1 and 2 , slightly improving the performance compared to the simple full average. Recall that those functionals are the most uncorrelated to each other. It should be noted that despite the identification rate did not increase significantly, the computational cost was considerably reduced since only 8 circus functions made the job of 21 of them, thanks to the feature selection analysis. As a result, we can conclude that redundant signatures may lead to deceiving similarity measures and overload the computational resources. Finally, the triangle-marked curve shows the identification performance of the orthonormal signatures. Note that further identification rate gains were achieved by minimizing the dependency among signatures, and thus enhancing their discriminative power. Also, it should be remarked that only 8 signatures were used instead of 21 without appealing to a feature selection analysis to pick up the best functional combination.

Having analyzed the performance of the orthonormal signatures in terms of information efficiency and identification rate, we can conclude that the proposed method provides three important advantages over the original method proposed by Petrou and Kadyrov [1]: (i) the method guarantees that all circus functions are only slightly correlated to each other, which provides saliency on each of them; (ii) if additional orthonormal signatures are necessary, there is no need to perform a feature selection analysis to verify that the new functionals retrieve uncorrelated information; (iii) the orthonormal signatures may achieve a higher identification performance than the traditional approach with a much lower number of signatures.

\section{CONCLUSIONS}

A novel method to derive orthonormal circus functions from the trace transform was proposed in this paper. A feature selection analysis of the traditional trace transform functionals revealed that most of them are highly correlated to each other. Consequently, most of these functionals retrieve descriptors that are inefficient in terms of the information conveyed as well as computationally expensive. In order to overcome these problems, we proposed a

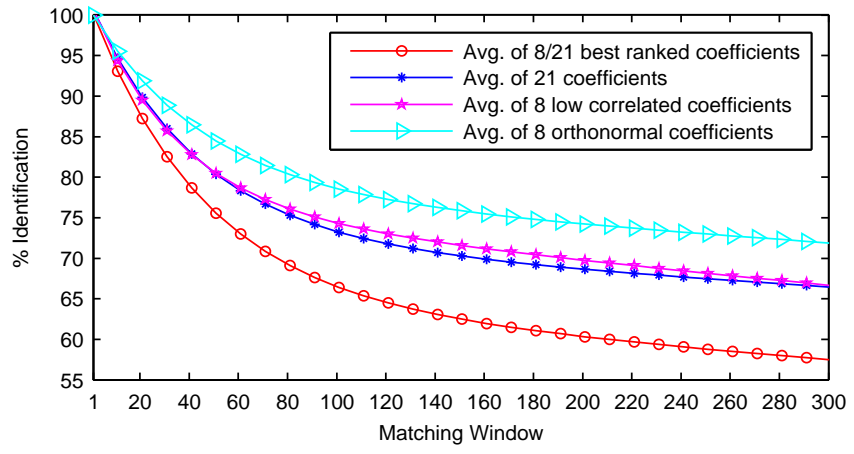

Fig. 4. Identification performance for different matching windows.

set of $P$-functionals based on Laguerre polynomials that, together with the orthonormalization of the sinogram, return non-redundant circus functions. In this way, we were able to reduce the number of signatures and maximize the information conveyed without compromising the identification performance.

\section{REFERENCES}

[1] M. Petrou and A. Kadyrov, "Affine invariant features from the trace transform," IEEE Transactions on Pattern Analysis and Machine Intelligence, vol. 26, pp. 30-44, 2004.

[2] S. Srisuk, M. Petrou, W. Kurutach, and A. Kadyrov, "A face authentication system using the trace transform," Pattern Analysis and Applications, vol. 8, pp. 50-61, 2005.

[3] W. Kurutach, R. Fooprateepsiri, and S. Phoomvuthisarn, "A highly robust approach face recognition using hausdorff-trace transformation," in Proc. of the 17th int. conf. on Neural information processing: models and applications (ICONIP'10), 2010, pp. 549-556.

[4] G. Goudelis, K. Karpouzis, and S. Kollias, "Robust human action recognition using history trace templates," in Proc. of the 12th Int. Workshop on Image Analysis for Multimedia Interactive Services (WIAMIS), 13-15 April, 2011.

[5] A. Kadyrov and M. Petrou, "The trace transform and its applications," IEEE Trans. Pattern Anal. Mach. Intell., vol. 23, pp. 811-828, August 2001.

[6] D. Zarpalas, P. Daras, A. Axenopoulos, D. Tzovaras, and M.G. Strintzis, "3d model search and retrieval using the spherical trace transform,” EURASIP J. Appl. Signal Process., vol. 2007, pp. 207-207, January 2007.

[7] A. Kadyrov and M. Petrou, "Affine parameter estimation from the trace transform," IEEE Trans. Pattern Anal. Mach. Intell., vol. 28, pp. 1631-1645, October 2006.

[8] S. Fahmy, C. Bouganis, P. Cheung, and W. Luk, "Real-time hardware acceleration of the trace transform," Journal of RealTime Image Processing, vol. 2, pp. 235-248, 2007.

[9] A. Kadyrov and M. Petrou, "Object signatures invariant to affine distortions derived from the trace transform," Image and Vision Computing, vol. 21, no. 13-14, pp. 1135 - 1143, 2003.

[10] H. Liu and L. Yu, "Toward integrating feature selection algorithms for classification and clustering," IEEE Trans. on Knowledge and Data Engineering, vol. 17, pp. 491-502, 2005.

[11] Peter Schönemann, "A generalized solution of the orthogonal procrustes problem," Psychometrika, vol. 31, pp. 1-10, 1966. 